\title{
Fiabilidad del navegador en la colocación de tornillos pediculares toracolumbares
}

\author{
S. Fuster*; A. Vega**; G. Barrios**; I. Urdaneta**; O. Ojeda**; M. Macchia**; A. Combalia**; J. Berenguer** y \\ T. Pujol \\ Unitat de Raquis. Hospital Clínic. Universitat de Barcelona.
}

Resumen

Introducción. La cirugía guiada por imagen es uno de los más importantes avances tecnológicos dentro de la cirugía del Raquis ya que permite al cirujano realizar una navegación multiplanar tridimensional en tiempo real en el interior de una vértebra.

Material y métodos. Realizamos un estudio clínico prospectivo no randomizado sobre la fiabilidad en la colocación de tornillos pediculares mediante un sistema de navegación optoelectrónico (SurgiGATE Spine 2.1 Medivision). Se estudiaron veintinueve pacientes intervenidos por diferentes patologías en columna toracolumbar incluyendo: degenerativas (54\%), espondilolistesis $(21 \%)$, fracturas (14\%), escoliosis (7\%) y espondilodiscitis (4\%). Un paciente fue eliminado del estudio debido a un fallo técnico en el equipo de navegación. Se obtuvieron imágenes de $\mathrm{TC}$ pre y postoperatorias de cada paciente y éstas fueron evaluadas por dos neurorradiólogos independientes. La colocación correcta se definió de acuerdo a la escala de Heary en 5 grados.

Resultados. Se colocaron 163 tornillos, 29 en la columna toracolumbar y 134 en la columna lumbosacra. Hemos conseguido una colocación totalmente intraósea (Grado I) en el $\mathbf{9 9 , 4 \%}$ de tornillos en la columna lumbosacra y en un $\mathbf{1 0 0 \%}$ en la columna toracolumbar. Se comprobó el error de colocación (Grado III) en un pedículo de L3 en la concavidad de una escoliosis. No se observaron complicaciones relacionadas con los implantes.

Conclusiones. El bajo porcentaje de tornillos mal colocados en este estudio se compara favorablemente con los resultados publicados en la literatura. Nuestros resultados indican que la cirugía guiada por imagen aplicada a la cirugía del raquis es una técnica segura para la fijación transpedicular.

PALABRAS CLAVE: Fiabilidad. Navegador. Tornillos pediculares. Toracolumbar.

Recibido: 30-07-09. Aceptado: 26-11-09
Accuracy of pedicle screw insertion in the thoracolumbar spine using image-guided navigation

Summary

Introduction. Computer image guidance is one of the most significant technologic advancements in the spine surgery, because preoperative or intraoperative images can be used for multiplanar, three-dimensional intraoperative navigation.

Material and methods. We performed a prospective clinical study to assess the accuracy of pedicle screw insertion using an optoelectronic navigation system (SurgiGaTE Spine 2.1 Medivision). The study population included 29 patients with diverse disorders of the thoraco- lumbar spine (degenerative 54\%, spondylolisthesis $21 \%$, fractures $14 \%$, scoliosis $7 \%$ and spondylodiscitis $4 \%$ ). One patient was excluded from the study because problems with the specific instruments or the computer system. Pre and post-operative axial computed tomography images were obtained for each patient and analyzed by two independent radiologists to placement accuracy. The correct location was defined accord to Heary scale in $\mathbf{5}$ grades.

Results. 163 image-guided thoraco-lumbar pedicle screws were placed 29 in the thoracolumbar spine and 134 in the lumbosacral spine. We achieved a completely intraosseous placement (Grade I) in $99.4 \%$ of lumbosacral spine screws and $100 \%$ of thoracolumbar spine screws. Only one misplaced screw (Grade III) in the pedicle of L III in the concavity of a scoliosis was reported. No implant related complications were noted.

Conclusions. The low rate of misplaced screws in this prospective study compares favorably with previously published results. Our initial results indicate that Image-guided spinal surgery is a safe technique which improves surgical performance during posterior transpedicle stabilization.

Abreviaturas. TC: tomografia computarizada. RM: resonancia magnetica. 2D: dos dimensiones. 
KEY WORDS. Accuracy. Navigation. Pedicle screw. Thoraco-lumbar spine.

\section{Introducción}

La cirugía guiada por imagen es uno de los más importantes avances tecnológicos dentro de la cirugía del Raquis ya que permite al cirujano realizar una navegación multiplanar tridimensional en tiempo real en el interior de una vértebra . Introducida en 1995, fue diseñada para aumentar la precisión durante la instrumentación espinal ${ }^{3,4,19,26}$. Una de las ventajas de la instrumentación en la cirugía de la columna vertebral es una mayor estabilidad biomecánica, con el consiguiente aumento de los índices de fusión. Sin embargo, dichas técnicas no se encuentran libres de complicaciones y éstas pueden ser graves e incluyen lesiones vasculares, viscerales y neurológicas.

El uso de tornillos pediculares en los procedimientos de fusión espinal ha ido en constante aumento durante la última década y la cirugía guiada por imagen se ha utilizado para mejorar la precisión y la seguridad en su colocación $^{9,10}$. Los trabajos comparativos entre Navegador y técnica convencional demuestran diferencias de error estadísticamente significativas favorables al navegador y estos errores aumentan en los pedículos críticos $(<9 \mathrm{~mm})$ o en anatomías alteradas como en escoliosis $6,7,7,15,16,25$.

Describimos nuestra experiencia con 168 tornillos pediculares toracolumbares colocados mediante un sistema de navegación como procedimiento auxiliar de una fusión espinal.

\section{Material y métodos}

Realizamos un estudio clínico prospectivo no randomizado en la Unidad de Raquis del Hospital Clinic de Barcelona sobre la fiabilidad en la colocación de tornillos pediculares mediante el uso de un sistema de navegación optoelectrónico (SurgiGATE Spine 2.1 Medivision). Se estudiaron veintinueve pacientes intervenidos quirúrgicamente de Noviembre del 2001 a Diciembre del 2002 por diferentes patologías en la columna toracolumbar y lumbosacra.

Mediante un protocolo preoperatorio de navegación raquídea se realiza un scanner de cortes finos $(2 \mathrm{~mm})$ incluyendo el segmento superior e inferior de los niveles afectados. Se transfieren las imágenes hacia la estación de trabajo del Vector Visión. Utilizando el modelo de la columna vertebral regenerada en la pantalla del ordenador se procede a realizar la planificación para definir los puntos de entrada y diana, así como los puntos de referencia anatómica en la apófisis espinosa y facetas articulares (derecha e izquierda, superior e inferior) del nivel correspondiente.

Técnica: abordaje lumbar posterior. Colocación del clamp referenciador en la apófisis espinosa de la vértebra a tratar. Con la ayuda del puntero electrónico se identifican los puntos anatómicos en espinosas y articulares y se confrontan con los datos de imagen almacenados preoperatoriamente. $\mathrm{Si}$ el margen de error no es superior a $2 \mathrm{~mm}$ se puede proceder a la navegación; sin embargo, si el margen de error es mayor se repite el procedimiento. Si persiste el error de confrontación o en anatomías alteradas por cirugías previas o anomalías congénitas se realiza un mapa de superficie mediante la adquisición de múltiples puntos anatómicos. Esta fase de confrontación se repite en cada nivel a tratar.

Hemos introducido los tornillos pediculares con navegación multiplanar tridimensional en tiempo real intentando mantener las trayectorias planeadas previamente. Se identifica el punto de entrada, ángulo de inserción y profundidad de inserción para cada tornillo pedicular.

Se obtuvieron imágenes de TC postoperatorias de cada paciente que fueron evaluadas por dos neurorradiólogos independientes siguiendo el mismo protocolo utilizado para el estudio preoperatorio. La colocación de cada tornillo pedicular se evaluó de acuerdo a la escala de Heary en 5 grados $^{18}$. Tabla 1.

Tabla 1

Escala tomográfica de Heary para evaluar la colocación del tornillo en relación al pedículo

\begin{tabular}{l|l}
\hline Grado & Descripción \\
\hline Grado I & Tornillo completamente contenido en el pedículo. \\
Grado II & $\begin{array}{l}\text { Tornillo viola la pared lateral del pedículo pero la punta del tornillo esta totalmente contenida en el cuerpo } \\
\text { vertebral. }\end{array}$ \\
Grado III & Tornillo perfora la pared lateral del pedículo. \\
Grado IV & Perforación de la pared medial o inferior del pedículo. \\
Grado V & Viola el pedículo o el cuerpo vertebral y pone en peligro la médula espinal, raíz nerviosa, o los grandes \\
& vasos y requiere de inmediata revisión \\
\hline
\end{tabular}





Un paciente fue eliminado del estudio debido a un fallo técnico en el equipo de navegación.

\section{Resultados}

De Noviembre del 2001 a Diciembre del 2002, 29 pacientes ( 9 hombres y 20 mujeres) fueron operados en la Unidad de Cirugía de Raquis del Hospital Clínic de Barcelona por patologías en columna toracolumbar y lumbar (T11 - S2) incluyendo: degenerativas (54\%), espondilolistesis $(21 \%)$, fracturas $(14 \%)$, escoliosis $(7 \%)$ y espondilodiscitis (4\%). Figuras 1 y 2. Todos los pacientes fueron sometidos a una fijación pedicular guiada por navegador como procedimiento auxiliar de una fusión espinal.

Se colocaron 163 tornillos, 29 en la columna toracolumbar (T11- L2). y 134 en la columna lumbosacra (L3 - S2) Figura 3. Conseguimos una colocación totalmente intraósea (Grado I) en 133 de $134(99,4 \%)$ tornillos de la columna lumbosacra y en 29 de $29(100 \%)$ tornillos de la

Figura 2. 1) Radiografia, 2) $R M$ y 3) $T C$ de paciente con enfermedad discal degenerativa L5-S1, 2 y 3) planeación preoperatoria de las trayectorias, 6) TC postoperatoria en corte axial, 7) coronal y 8) sagital en la cual se observan los tornillos contenidos totalmente en el pedículo correspondiente. 


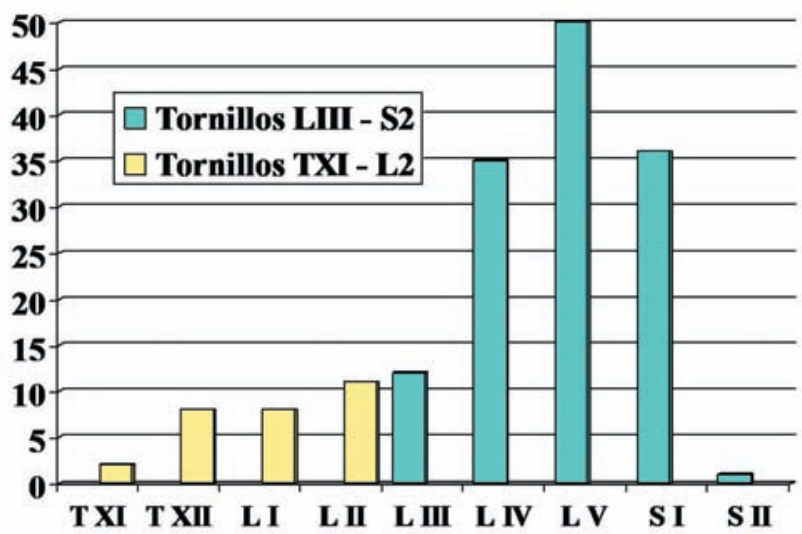

Figura 3. Distribución de los tornillos pediculares colocados en la columna toracolumbar (T11-L2) y lumbosacra (L3-S2).

columna toracolumbar. Se identificó un tornillo mal colocado (Grado III) en el pedículo izquierdo de L3 en la concavidad de una escoliosis. No se observaron complicaciones relacionadas con los implantes.

\section{Discusión}

El uso de la fijación transpedicular para la estabilización de la columna vertebral es una técnica indicada en infecciones, tumores, trauma y deformidades ${ }^{1,20}$. El método tradicional para la colocación de tornillos pediculares utiliza referencias anatómicas de superficie combinado con la orientación que proporciona la fluoroscopía peroperatoria. Aunque esta técnica ha demostrado ser útil, tiene limitaciones, ya que solamente proporciona una imagen en dos dimensiones de una compleja estructura tridimensional. Con el uso de esta técnica diferentes autores reportan errores por malposición de los tornillos en porcentajes que oscilan entre un 10 a un $20 \%$ y que comportan lesión neurológica en el $4.5 \%$ de los pacientes ${ }^{14,27,32}$. En alguna ocasión, como refiere Gómez de la Riva y cols. sobre una serie de 74 pacientes con estenosis de canal lumbar intervenidos mediante descompresión y fijación pedicular, la malposición de los tornillos pediculares acompañado de clínica neurológica obliga a la reintervención quirúrgica en un $2,7 \%$ de los pacientes ${ }^{17}$.

La colocación de tornillos pediculares en la columna cervical y torácica es técnicamente más demandante para el cirujano que en la columna lumbar. La razón es que los pedículos en estas regiones son más pequeños y presentan un alto grado de variabilidad en cada paciente ${ }^{20,30}$.

Dependiendo de la técnica de inserción del tornillo pedicular (laminar abierto, Steffee's, o Roy-Camille) y los criterios utilizados para determinar la adecuada colocación de los tornillos dentro del pedículo, la tasa de malposición de tornillos pediculares va desde un 3 a un $55 \%$ en diversas series $^{8,14,28,31,34}$.

Se ha documentado que la mala posición de un tornillo pedicular en una fijación mono segmentaria (4 tornillos) compromete la estabilidad de la fijación vertebral porque disminuye la fuerza de agarre hasta en un $11 \%{ }^{13}$. El porcentaje aumenta en función del número de tornillos mal colocados.

Numerosas técnicas han sido descritas para intentar mejorar la precisión en la colocación de tornillos pediculares: visión directa mediante laminectomía, monitorización neurofisiológica durante la introducción del tornillo en el pedículo y la endoscopia intrapedicular o epidural ${ }^{5,11}$.

Diferentes estudios realizados tanto in vivo como in vitro indican que la cirugía guiada por imagen mejora de forma significativa la colocación correcta de los tornillos dentro del pedículo cuando se compara con el método tradicional $2,21,22,24,26$.

La cirugía asistida por ordenador mejora la precisión durante la fijación transpedicular y minimiza el riesgo de lesiones neurológicas y vasculares. Además de reducir las posibles complicaciones, existe una ventaja adicional ya que la colocación interósea de cada tornillo proporciona una mejor fuerza de agarre por cada nivel instrumentado.

La cirugía guiada por imagen de TC proporciona una anatomía precisa del pedículo y reduce la exposición a la radiación del paciente y del equipo quirúrgico, pero requiere de una curva de aprendizaje y una preparación preoperatoria que incluye la tomografía computarizada con un protocolo específico, la adquisición y transferencia de datos y el registro de pacientes. Un estudio in vitro reveló que el promedio de tiempo que lleva la inserción de un tornillo pedicular mediante navegación fue de 13,5 minutos, frente a 4 minutos del grupo que usó fluoroscopia lateral. La tasa de violación de la pared medial del pedículo que puede causar lesiones neurológicas catastróficas es del $0 \%$ en comparación con el 5\% en el grupo de fluoroscopia ${ }^{3}$. En nuestra experiencia el tiempo total de la intervención quirúrgica disminuye conforme el cirujano se familiariza con la técnica de navegación.

El desarrollo de sistemas de fluoronavegación en 2 ó 3 dimensiones aparece para hacer frente a las cuestiones desfavorables de la cirugía guiada por imagen de $\mathrm{TC}^{12,23,33}$. El equipo no requiere de un registro previo, se reduce el tiempo y dosis de radiación, se evitan movimientos repetidos del fluoroscopio durante la cirugía ya que la visualización de los instrumentos quirúrgicos en relación con la anatomía del paciente se determina desde el principio de la cirugía.

Respecto al punto de comparación de ambos sistemas de navegación Tian y colaboradores tras un metanálisis de 54 
estudios (35 in vivo y 20 in vitro) concluyen que la navegación basada en imagen de TC proporciona una mayor precisión en la colocación de tornillos pediculares en ambos grupos (in vivo $=90.76 \%$, in vitro $=94.59 \%$ ) comparado con el grupo de fluoronavegación $2 \mathrm{D}$ (in vivo $=85.48 \%$, in vitro $=90.12 \%$ ). En nuestro grupo de estudio, utilizando un sistema de navegación de primera generación guiado por imagen de TC, conseguimos una fiabilidad del $99,4 \%$ en la inserción de tornillos pediculares, lo que se corresponde con los resultados de las series publicadas en el metaanálisis de $\operatorname{Tian}^{29}$.

Existen diversas condiciones que contraindican la cirugía guiada por imágenes como las patologías con inestabilidad o ausencia del arco posterior, ya que inducen a un error de los sistemas de medición; en tales circunstancias los autores utilizan el método habitual mediante control fluoroscópico. En nuestra opinión la utilización frecuente de la cirugía guiada por imagen no debe perjudicar la habilidad de los cirujanos para realizar la técnica convencional.

\section{Conclusiones}

El bajo porcentaje de tornillos mal colocados $(0,06 \%)$ en este estudio se compara favorablemente con los resultados publicados previamente en la literatura.

Aunque el número de pacientes en el presente estudio es pequeño, los resultados son alentadores ya que indican que la cirugía guiada por imagen aplicada a la cirugía del raquis es una técnica fiable y segura durante la fijación transpedicular.

\section{Nota}

Trabajo aceptado y presentado como E-póster en el IX Congreso de la Sociedad Española de Neurorraquis. Barcelona 2009.

\section{Bibliografía}

1. Albert, T.J., Klein, G.R., Joffe, D., Vaccaro, A.R.: Use of cervicothoracic junction pedicle screws for reconstruction of complex cervical spine pathology. Spine 1998; 23: 15961599.

2. Amiot, L.P., Lang, K., Putzier, M., Zippel, H., Labelle, H.: Comparative results between conventional and computerassisted pedicle screw installation in the thoracic, lumbar, and sacral spine. Spine 2000; 25: 606-614.

3. Assaker, R., Reyns, N., Vinchon, M., Demondion, X., Louis, E.: Transpedicular screw placement: image-guided versus lateral view Fluoro scopy: in vitro simulation. Spine 2001; 26: 2160-2164.

4. Austin, M.S., Vaccaro, A.R., Brislin, B., Nachwalter, R., Hilibrand, A.S., Albert, T.J.: Image-guided spine surgery: a cadaver study comparing conventional open laminoforaminotomy and two image-guided techniques for pedicle screw placement in posterolateral fusion and nonfusion models. Spine 2002; 27: 2503-2508.

5. Clements, D.H., et al.: Evoked and spontaneous electromyography to evaluate lumbosacral pedicle screw placement. Spine 1996; 21: 600-604.

6. Davne, S.H., Myers, D.L.: Complications of lumbar spinal fusion with transpedicular instrumentation. Spine 1992; 17: S184-S189.

7. De la Torre-Gutiérrez, M., Martínez-Quiñones, J.V., Escobar-Solís, R., de la Torre-Gutiérrez: Spinal neuronavigation. Our experience. Neurocirugía 2001; 12: 490-498.

8. Esses, S.I., Sachs, B.L., Dreyzin, V.: Complications associated with the technique of pedicle screw fixation. A selected survey of ABS members. Spine 1993; 18: 22312239.

9. Fischgrund, J.S., Mackay, M., Herkowitz, H.N., Brower, R., Montgomery, D.M., Kurz, L.T.: 1997 Volvo Award winner in clinical studies. Degenerative lumbar spondylolisthesis with spinal stenosis: a prospective, randomized study comparing decompressive laminectomy and arthrodesis with and without spinal instrumentation. Spine1997; 22: 2807-2812.

10. France, J.C., Yaszemski, M.J., Lauerman, W.C., Cain, J.E., Glover, J.M., Lawson, K.J., et al.: A randomized prospective study of posterolateral lumbar fusion. Outcomes with and without pedicle screw instrumentation. Spine 1999; 24: 553-560.

11. Frank, E.H., Chamberland, D.L.: An endoscopic pedicle probe: Preliminary development. Neurol Res 1997; 6: 657561.

12. Fu, T.S., Wong, C.B., Tsai, T.T., Liang, Y.C., Chen, L.H., Chen, W.J.: Pedicle screw insertion: computed tomography versus fluoroscopic image guidance. Int Orthop 2008; 32: 517-521.

13. George, D.C., Krag, M.H., Johnson, C.C., Van Hal, M.E., Haugh, L.D., Grobler, L.J.: Hole preparation techniques for transpedicle screws: Effect on pull-out strenght from human cadaveric vertebrae. Spine 1991; 16: 181-184.

14. Gertzbein, S.D., Robbins, S.E.: Accuracy of pedicular screw placement in vivo. Spine 1990; 15: 11-14.

15. Gluf, W.M., Schmidt, M.H., Apfelbaum, R.I.: Atlantoaxial transarticular screw fixation: a review of surgical indications, fusion rate, complications, and lessons learned in 191 adult patients. J Neurosurg Spine 2005; 2: 155-163.

16. Gluf, W.M., Brockmeyer, D.L.: Atlantoaxial transarticular screw fixation: a review of surgical indications, fusion rate, complications, and lessons learned in 67 pediatric patients. J Neurosurg. Spine 2005; 2: 164-169.

17. Gómez de la Riva, A., Isla, A., Pérez-López, C., Ortega, R., Fernández-Miranda, J.C., Heredero, J.: Causes of reoperations in patients with lumbar spinal stenosis treated with instrumentations. Neurocirugía 2006; 17: 232-239. 
18. Heary, R.F., Bono, C.M., Black, M.: Thoracic pedicle screws: postoperative computerized tomography scanning assessment. J Neurosurg 2004; 100 (4 Suppl Spine): 325-331.

19. Kalfas, I.H., Kormos, D.W., Murphy, M.A., McKenzie, R.L., Barnett, G.H., Bell, G.R., et al.: Application of frameless stereotaxy to pedicle screw fixation of the spine. J Neurosurg 1995; 83: 641-647.

20. Krag, M.H., Weaver, D.L., Beynnon, B.D., Haugh, L.D.: Morphometry of the thoracic and lumbar spine related to transpedicular screw placement for surgical spinal fixation. Spine 1988; 13: 27-32.

21. Laine, T., Schlenzka, D., Mäkitalo, K., Tallroth, K., Nolte, L.P., Visarius, H.: Improved accuracy of pedicle screw insertion with computer-assisted surgery. A prospective clinical trial of 30 patients. Spine 1997 22: 1254-1258.

22. Laine, T., Lund, T., Ylikoski, M., Lohikoski, J., Schlenzka, D.: Accuracy of pedicle screw insertion with and without computer assistance: a randomised controlled clinical study in 100 consecutive patients. Eur Spine J 2000; 9: 235-240.

23. Lekovic, G.P., Potts, E.A., Karahalios, D.G., Hall, G.: A comparison of two techniques in image-guided thoracic pedicle screw placement: a retrospective study of 37 patients and 277 pedicle screws. J Neurosurg Spine 2007; 7: 393-398.

24. Ludwig, S.C., Kowalski, J.M., Edwards, C.C. $2^{\text {nd }}$, Heller, J.G.: Cervical pedicle screws: comparative accuracy of two insertion techniques. Spine 2000; 25: 2675-2681.

25. Matsuzaki, H., Tokuhashi, Y., Matsumoto, F., Hoshino, M., Kiuchi, T., Toriyama, S.: Problems and solutions of pedicle screw plate fixation of lumbar spine. Spine 1990; 15 : 1159-1165.

26. Rajasekaran, S., Vidyadhara, S., Ramesh, P., Shetty, A.P.: Randomized clinical study to compare the accuracy of navigated and non-navigated thoracic pedicle screws in deformity correction surgeries. Spine 2007; 32: 56-64.

27. Roy-Camille, R., Saillant, G., Mazel, C.: Internal fixation of the lumbar spine with pedicular plating. Clin Orthop Relat Res 1986; 203: 7-17.

28. Sapkas, G.S., Papadakis, S.A., Stathakopoulos, D.P., Papagelopoulos, P.J., Badekas, A.C., Kaiser, J.H.: Evaluation of pedicle screw position in thoracic and lumbar spine fixation using plain radiographs and computed tomography. A prospective study of 35 patients. Spine 1999; 24: 1926-1929.

29. Tian, N.F., Xu, H.Z.: Image-guided pedicle screw insertion accuracy: a meta-analysis. Int Orthop. 2009; 33: 895-903.

30. Vaccaro, A.R., Rizzolo, S.J., Allardyce, T.J., Ramsey, M., Salvo, J., Balderston, R.A., et al.: Placement of pedicle screws in the thoracic spine. Part I. Morphometric analysis of the thoracic vertebrae. J Bone Joint Surg Am 1995; 77: 11931199.

31. Vaccaro, A.R., Rizzolo, S.J., Balderston, R.A., Allardyce, T.J., Garfin, S.R., Dolinskas, C., et al.: Placement of pedicle screws in the thoracic spine. Part II: An anatomical and radiographic assessment. J Bone Joint Surg Am 1995; 77: 1200-1206.

32. Weinstein, J.N., Spratt, K.F., Spengler, D., Brick, C., Reid, S.: Spinal pedicle fixation: reliability and validity of roentgenogram-based assessment and surgical factors on successful screw placement. Spine 1988; 13: 1012-1018.

33. Wendl, K., von Recum, J., Wentzensen, A., Grützner, P.A.: Iso-C (3D0-assisted) navigated implantation of pedicle screws in thoracic lumbar vertebrae. Unfallchirurg 106:907913.

34. Xu, R., Ebraheim, N.A., Ou, Y., Yeasting, R.A.: Anatomic considerations of pedicle screw placement in the thoracic spine. Roy-Camille technique versus open-laminar technique. Spine 1998; 23: 1065-1068.

Fuster, S.; Vega, A.; Barrios, G.; Urdaneta,I.; Ojeda,O.; Macchia, M.; Combalia, A.; Berenguer, J.; Pujol, T.: Fiabilidad del navegador en la colocación de tornillos pediculares toracolumbares. Neurocirugía 2010; 21: 306-311.

Correspondencia: Dr. Salvador Fuster Obregón. Unitat de Raquis. Hospital Clínic. Universitat de Barcelona. Villarroel 170, Escala 12, planta 0. 08036 Barcelona. España.

E-mail:sfuster@clinic.ub.es 